\title{
Myometrial invasion and overall staging of endometrial carcinoma: assessment using fusion of T2-weighted magnetic resonance imaging and diffusion-weighted magnetic resonance imaging
}

This article was published in the following Dove Press journal:

OncoTargets and Therapy

\section{Yu Guo, ${ }^{1,2}$ Ping Wang, ${ }^{2}$ Penghui Wang, ${ }^{2}$ Wei Gao, Fenge $\mathrm{Li}^{3}{ }^{3}$ Xueling Yang, Hongyan $\mathrm{Ni}^{2}{ }^{2}$ Wen Shen, ${ }^{2}$ Zhi Guo' \\ 'Department of Interventional Therapy, Tianjin Medical University Cancer Institute and Hospital, National Clinical Research Center for Cancer, Key Laboratory of Cancer Prevention and Therapy, Tianjin, Tianjin's Clinical Research Center for Cancer, Tianjin, ${ }^{2}$ Department of Radiology, Tianjin First Center Hospital, The First Central Clinical College of Tianjin Medical University, Tianjin, ${ }^{3}$ Department of Gynecology, Tianjin First Center Hospital, Tianjin, People's Republic of China}

Correspondence: Zhi Guo Department of Interventional Therapy, Tianjin Medical University Cancer Institute and Hospital, National Clinical Research Center for Cancer, Key Laboratory of Cancer Prevention and Therapy, Tianjin, Tianjin's Clinical Research Center for Cancer, Binshui Road, Hexi Qu, Tianjin 300060, People's Republic of China

Tel +8622 23340123

Email guozhil955@163.com

Wen Shen

Department of Radiology, Tianjin First Center Hospital, The First Central Clinical College of Tianjin Medical University, 24 Fukang Rd, Nankai Qu, Tianjin 300192, People's Republic of China Tel +8622 23626583

Email shenwen66happy@163.com
Background: The age of onset of endometrial carcinoma has been decreasing in recent years. In endometrial carcinoma, it is important to accurately assess invasion depth and preoperative staging. Fusion of T2-weighted magnetic resonance imaging (T2WI) and diffusion-weighted magnetic resonance imaging (DWI) may contribute to the improvement of anatomical localization of lesions.

Materials and methods: In our study, a total of 58 endometrial carcinoma cases were included. Based on the revised 2009 International Federation of Gynecology and Obstetrics staging system, a fusion of T2WI and DWI was utilized for the evaluation of invasion depth and determination of the overall stage. Postoperative pathologic assessment was considered as the reference standard. The consistency of T2WI image staging and pathologic staging, and the consistency of fused T2WI and DWI and pathologic staging were all analyzed using Kappa statistics.

Results: Compared with the T2WI group, a significantly higher diagnostic accuracy was observed for myometrial invasion with fusion of T2WI and DWI $(77.6 \%$ for T2WI; $94.8 \%$ for T2WI-DWI). For the identification of deep invasion, we calculated values for diagnostic sensitivity (69.2\% for T2WI; $92.3 \%$ for T2WI-DWI), specificity ( $80 \%$ for T2WI; $95.6 \%$ for T2WI-DWI), positive predictive value (50\% for T2WI; $85.7 \%$ for T2WI-DWI), and negative predictive value $(90 \%$ for T2WI; $97.7 \%$ for T2WI-DWI). In summary, T2WI-DWI fusion exhibits higher diagnostic accuracy with respect to staging relative to T2WI only $(81.0 \%$ for T2WI; 94.8\% for T2WI-DWI).

Conclusion: Fused T2WI-DWI may represent a noninvasive, lower cost approach for the effective assessment of myometrial invasion and staging of endometrial carcinoma.

Keywords: endometrial carcinoma, T2WI, DWI, myometrial invasion, magnetic resonance imaging, fusion, staging

\section{Introduction}

A 5-year survival rate of $96 \%$ has been reported for stage I endometrial carcinoma patients, and $17 \%$ for stage IV patients. ${ }^{1}$ About $75 \%$ of endometrial cancer patients are in stage I when they start their treatments. ${ }^{2}$ Laparoscopic surgery and conservative hormone treatment are recent options in the treatment of early-stage endometrial cancer cases, ${ }^{3-5}$ although some investigators have advocated another surgical method, surgical lymphadenectomy, in cases of deep myometrial invasion. ${ }^{6-8}$ Accurate assessment of the myometrial invasion depth and endometrial cancer staging prior to surgery is very important for patients and definitely affect treatment planning and subsequent prognosis. ${ }^{9-12}$ In 2009, the Committee of the International Federation of Gynecology 
and Obstetrics (FIGO) revised the FIGO staging system (1988 version) for endometrial carcinoma, providing a better treatment basis for endometrial carcinoma. ${ }^{13,14}$

Magnetic resonance imaging (MRI) is a more accurate approach for the assessment of endometrial carcinoma staging compared with ultrasonography and CT before surgery. ${ }^{15-19}$ In fact, many studies have shown that dynamic contrast-enhanced MRI (DCE-MRI) is a good choice of imaging modality for predicting invasion depth. However, the role of DCE-MRI in overall staging is still controversial, and some researchers have suggested that there was no benefit. ${ }^{20-22}$ A retrospective study found that DCE-MRI did not improve diagnostic performance relative to viewing T2-weighted imaging (T2WI) independently with respect to predicting invasion depth, ${ }^{23}$ and DCE-MRI - depending upon the contrast agent used - may involve greater costs and risks. As for endometrial carcinoma, some studies have shown that diffusion-weighted imaging (DWI) exhibits better performance than DCE-MRI in assessing myometrial invasion. ${ }^{24-26}$ Therefore, it is necessary to find a simpler and more cost-effective imaging modality.

The combination of T2WI and DWI fuses morphologic and functional observations, which can improve the anatomical localization of lesions, and the entire procedure takes $<30$ s. Fused images possess many other advantages, such as simplicity of operation, intuitiveness of images, and noninvasiveness, and the combination of T2WI and DWI has been used in many studies. ${ }^{27-30}$ Reports have shown that the fused images are a very good modality for displaying anatomical structure and functional information, and improve the accuracy of diagnosis. The high accuracy of fused T2WIDWI images has been useful in assessing locally recurrent pelvic malignancies. ${ }^{27}$

No data are currently available about the use of fused T2WI-DWI in assessing invasion depth and stage of endometrial carcinoma. Hence, in this study, we aim to investigate the performance of 3T fused T2WI-DWI $\left(b=1,000 \mathrm{~s} / \mathrm{mm}^{2}\right)$ prior to surgery, which could lessen the overall patient burden.

\section{Materials and methods \\ Cases}

We obtained the approval of the Tianjin First Center Hospital Medical Ethics Committee and received informed consent from enrolled cases. Fifty-eight endometrial carcinoma patients treated at our hospital from April 2013 to January 2016 were included in this investigation. The average patient age was $60.41 \pm 7.75$ years (range, 45-78 years). No patient had previously received hormone replacement therapy. A total of 58 cases were enrolled, based on our inclusion criteria of:
1) the diagnosis of endometrial carcinoma being confirmed by biopsy after surgery; 2) patients having not received any other surgical or non-surgical treatments; 3) completion of the MRI examination before surgery, including conventional unenhanced MRI and DWI examination; 4) the time interval between surgery and MR examination being $<2$ weeks; and $5)$ the presence of detailed clinical medical records.

\section{MRI}

All MRI examinations were conducted using a 3T MRI system (Trio Tim; Siemens Medical Systems, Erlangen, Germany), 1-2 weeks prior to surgery. A 32-channel, phased array body coil was utilized. Relative parameters were as follows: axial T1WI (repetition time $[\mathrm{TR}]=630 \mathrm{~ms}$, echo time $[\mathrm{TE}]=11 \mathrm{~ms}$, field of view $[\mathrm{FOV}]=200 \times 200 \mathrm{~mm}$, matrix $=256 \times 320$, number of excitation $[\mathrm{NEX}]=3,23$ scanning layers); axial T2WI $(\mathrm{TR}=4,000 \mathrm{~ms}, \mathrm{TE}=101 \mathrm{~ms}, \mathrm{FOV}=200 \times 200 \mathrm{~mm}$, matrix $=256 \times 320, \mathrm{NEX}=3,23$ scanning layers); sagittal and coronal T2WI with fat suppression sequence $(\mathrm{TR}=4,000 \mathrm{~ms}$, $\mathrm{TE}=92 \mathrm{~ms}, \mathrm{FOV}=200 \times 200 \mathrm{~mm}$, matrix $=256 \times 320$, NEX $=3$ ). Axial DWI data were obtained through a singleshot, echo-planar technique, with $\mathrm{TR}=5,000 \mathrm{~ms}, \mathrm{TE}=81 \mathrm{~ms}$, matrix $=128 \times 128, \mathrm{NEX}=3$, and FOV $=200 \times 200 \mathrm{~mm}$; the diffusion sensitive factor $b$-value was $1,000 \mathrm{~s} / \mathrm{mm}^{2}$. Sequences were performed with identical section thickness and a gap in axial planes. This study was performed with normal respiration.

\section{Image analysis}

One radiologist, with 15 years of experience in gynecologic radiology, read images independently without the knowledge of clinical and pathologic information; and MRI images were reviewed in random order during the diagnostic process. We used image processing software on a MR post-processing workstation (Siemens Syngo 2010A). Myometrial invasion and staging were analyzed using T2WI and fused T2WIDWI. The radiologist assessed the following parameters: 1) invasion depth, 2) cervical stromal involvement, 3) bilateral adnexa and vaginal or other pelvic organ involvement, 4) presence of enlarged lymph nodes, and 5) presence of distant metastatic disease. The invasion depth was defined as the distance between the inner myometrial interface and the invasion point. We categorized invasion depth of $<50 \%$ of the myometrial thickness as superficial invasion, and extension $\geq 50 \%$ of the myometrium as deep invasion.

\section{Histologic analysis}

All patients were treated surgically, and the time interval between MR examination and surgery was $<2$ weeks. Histopathologic characteristics of the lesions were observed by 
an experienced pathologist, and the following were evaluated: uterine myometrial invasion; presence or absence of cervical stromal involvement; complete or incomplete serous membrane; vaginal and parametrial involvement; and lymph node metastasis and distant metastasis. These factors are in accordance with 2009 revised FIGO staging system for postoperative pathologic staging.

\section{Statistical analysis}

Statistical analysis software (IBM SPSS Statistics for Windows, version 19.0; IBM Corp., Armonk, NY, USA) was used for data processing. To analyze the infiltration depth, we calculated data accuracy, sensitivity, specificity, and positive and negative predictive values using T2WI and fused T2WI-DWI. Accuracy comparisons between T2WI and fused T2WI-DWI images were performed via $\chi^{2}$ test. The consistency of T2WI images staging and pathologic staging, and the consistency of fused T2WI-DWI images staging and pathologic staging were all analyzed using Kappa statistics. The criteria for the Kappa statistics were $>0.75$ (excellent agreement) and $<0.4$ (poor agreement). A $P$-value $<0.05$ was considered to show a statistically significant difference between the groups.

\section{Results}

\section{Histopathologic diagnoses}

Histopathologic diagnoses are listed in Table 1. Of 58 endometrial carcinoma cases, postoperative histologic assessment revealed 51 cases with endometrioid adenocarcinoma, 2 cases with clear-cell differentiation, 1 case with adenocarcinoma and papillary differentiation, and 4 cases with a mixed pattern. In addition, there were 43 cases with stage IA endometrial carcinoma; 8 cases with stage IB, 3 cases with stage II, and 4 cases with stage III. Forty-five cases were classified

Table I Surgical histopathologic characters

\begin{tabular}{|c|c|c|}
\hline & Data & Percentage (\%) \\
\hline \multicolumn{3}{|l|}{ Overall FIGO stage } \\
\hline IA & 43 & 74.I (43/58) \\
\hline IB & 8 & I $3.8(8 / 58)$ \\
\hline II & 3 & $5.17(3 / 58)$ \\
\hline III & 4 & $6.90(4 / 58)$ \\
\hline \multicolumn{3}{|l|}{ Myometrial invasion } \\
\hline Superficial & 45 & $77.6(45 / 58)$ \\
\hline Deep & 13 & $22.4(13 / 58)$ \\
\hline \multicolumn{3}{|l|}{ Histologic subtype } \\
\hline Endometrioid & 51 & $87.9(5 \mathrm{I} / 58)$ \\
\hline Clear cell & 2 & $3.4(2 / 58)$ \\
\hline Serous papillary & $\mathrm{I}$ & I.7 (I/58) \\
\hline Mixed & 4 & $6.9(4 / 58)$ \\
\hline
\end{tabular}

Abbreviation: FIGO, International Federation of Gynecology and Obstetrics. as having superficial invasion and 13 cases as deep invasion. None had lymph node metastasis or local spread of cancer upon histopathologic examination.

\section{Assessment of depth of invasion}

Fused T2WI-DWI images showed obvious boundaries of lesions, extent of involvement, and a clear contrast with surrounding normal structures. Pathology confirmed 45 cases with superficial invasion and 13 cases with deep invasion. Superficial invasion was determined correctly in 36 cases (accuracy rate of 80\%) and deep invasion in 9 cases (accuracy rate of $69.2 \%$ ) with T2WI. The accuracy of overall depth of invasion was $77.6 \%$ (45/58) with T2WI; 13 cases were wrongly diagnosed, including 9 cases of superficial invasion that were mistaken for deep myometrial invasion, and 4 cases of deep myometrial invasion that were mistaken for superficial myometrial invasion. Superficial myometrial invasion was correctly determined in 43 cases (accuracy of 95.6\%) and deep myometrial invasion in 12 cases (accuracy of $92.3 \%$ ) with T2WI-DWI fused images. The accuracy of overall depth of myometrial invasion was determined at $94.8 \%$ (55/58); 3 cases were misdiagnosed, including 2 cases of superficial myometrial invasion that were mistaken for deep invasion, and 1 case of deep invasion that was mistaken for superficial invasion. The accuracy of the assessment of myometrial invasion with these 2 methods is compared in Table 2. Fused T2WI-DWI images had higher diagnostic accuracy, sensitivity, specificity, and positive and negative predictive values compared with T2WI (Table 2).

\section{Staging of endometrial carcinoma}

For overall staging, 51 patients had tumors confined to the uterus, 3 patients had cervical stromal invasion, 4 patients had tumor invasion into serosa or adnexa, and no patient had enlarged lymph nodes or the presence of distant metastatic disease. We correctly staged 47 patients (81\%) with endometrial carcinoma using T2WI. Of patients who were incorrectly

Table 2 Sensitivity, specificity, and positive and negative predictive values for diagnosis of the depth of myometrial invasion with $\mathrm{T} 2 \mathrm{WI}$ and $\mathrm{T} 2 \mathrm{WI}-\mathrm{DWI}$

\begin{tabular}{lllll}
\hline Diagnosis & $\begin{array}{l}\text { Sensitivity } \\
\text { (\%) }\end{array}$ & $\begin{array}{l}\text { Specificity } \\
\text { (\%) }\end{array}$ & $\begin{array}{l}\text { Positive } \\
\text { predictive } \\
\text { value (\%) }\end{array}$ & $\begin{array}{l}\text { Negative } \\
\text { predictive } \\
\text { value (\%) }\end{array}$ \\
\hline $\begin{array}{l}\text { Superficial invasion } \\
\text { T2WI }\end{array}$ & $80(36 / 45)$ & $69.2(9 / 13)$ & $90(36 / 40)$ & $50(9 / 18)$ \\
T2WI-DWI & $95.6(43 / 45)$ & $92.3(12 / 13)$ & $97.7(43 / 44)$ & $85.7(12 / 14)$ \\
$\begin{array}{c}\text { Deep invasion } \\
\text { T2WI }\end{array}$ & $69.2(9 / 13)$ & $80(36 / 45)$ & $50(9 / 18)$ & $90(36 / 40)$ \\
T2WI-DWI & $92.3(12 / 13)$ & $95.6(43 / 45)$ & $85.7(12 / 14)$ & $97.7(43 / 44)$ \\
\hline
\end{tabular}

Abbreviations: DWI, diffusion-weighted imaging; T2WI, T2-weighted imaging. 
Table 3 Endometrial carcinoma staging with $\mathrm{T} 2 \mathrm{WI}$ and fused T2WI-DWI images compared with pathologic results

\begin{tabular}{lllll}
\hline T2WII & \multicolumn{4}{l}{ Pathologic diagnoses } \\
\cline { 2 - 5 } T2WI-DWI & IA & IB & II & III \\
\hline IA & $35 / 4$ I & $3 / I$ & $0 / 0$ & $0 / 0$ \\
IB & $8 / 2$ & $5 / 7$ & $0 / 0$ & $0 / 0$ \\
II & $0 / 0$ & $0 / 0$ & $3 / 3$ & $0 / 0$ \\
III & $0 / 0$ & $0 / 0$ & $0 / 0$ & $4 / 4$ \\
\hline
\end{tabular}

Abbreviations: DWI, diffusion-weighted imaging; T2WI, T2-weighted imaging.

staged, 8 cases were over-staged (stage IA mistaken for stage IB) and 3 cases were under-staged (stage IB for stage IA). We correctly staged 55 patients (94.8\%) with endometrial carcinoma using fused T2-weighted and DW images. Two cases were over-staged (stage IA for stage IB) and 1 case was understaged (stage IB for stage IA). The accuracy rate for staging endometrial carcinoma stage I was $78.4 \%$ (40/51) with T2WI and $94.1 \%(48 / 51)$ with fused imaging. The overall staging accuracy rate was $81 \%(47 / 58)$ with T2WI and $94.8 \%(55 / 58)$ with fused imaging (Table 3; Figure 1). In stage I endometrial carcinoma cases, 8 cases that were wrongly diagnosed with T2WI were correctly diagnosed with T2WI-DWI fused images (Figures 2 and 3). Therefore, fused T2WI-DWI images exhibited higher diagnostic accuracy than T2WI in the assessment of tumor staging. Statistical analyses revealed that results of endometrial carcinoma staging obtained from fused images and pathologic findings were more consistent than those obtained from T2WI images and pathologic findings (Table 4).

\section{Discussion}

We demonstrated that fused T2WI-DWI images were acceptable for assessing invasion depth, and significantly improved the staging of endometrial carcinoma. Our study illustrated that fused T2WI-DWI images showed obvious lesion boundaries, the extent of invasion, and depicted a clear contrast with the surrounding normal structures. This modality is therefore advantageous for the detection, localization, and assessment of invasion depth and tumor staging.
The sensitivity, specificity, and accuracy of fused T2WIDWI in evaluating superficial myometrial invading lesions were $95.5 \%, 92.3 \%$, and $95.6 \%$, respectively; for evaluating deep myometrial invading lesions, the sensitivity, specificity, and accuracy of fused T2WI-DWI were $92.3 \%, 95.5 \%$, and $92.3 \%$, respectively. These values were higher than the results for T2WI. The diagnostic accuracy rate of evaluating invasion depth using T2WI was high and consistent with previous reports in the literature..$^{14,31,32}$ To our knowledge, investigators who used fused T2-weighted and DW images for evaluation did not report overall staging of endometrial cancer (2009 stage). In this study, with fused T2-weighted and DW images, we diagnosed the overall staging of 58 patients with an accuracy rate of $94.8 \%(55 / 58)$, which was better than T2WI, particularly with respect to stage I endometrial carcinoma. The accuracy for stage IA and IB were $95.3 \%$ and $87.5 \%$, respectively, which was also significantly higher than T2WI. The fused images manifested higher diagnostic consistency and correlated well with pathologic findings. There are some reasons, however, for misjudgments (ie, overstaging and understaging). The lesions appear as significantly higher signals than muscles, which generally can be diagnosed on T2WI only, but it is relatively difficult to diagnose the lesions when they appear as iso-intense or the contrast signal is not obvious; this can lead to misdiagnosis or stage misjudgment (such as in Figure 3). In addition, interpretation of staging is compromised with concomitant conditions on MR images, such as tumor at cornus uteri, fibroid, or adenomyosis - these can interfere with the assessment of the lesions and easily lead to misdiagnosis. Fused T2-weighted and DW images, therefore, exhibit distinct advantages in diagnosing these lesions.

Extensive research has shown that DCE-MRI is an imaging modality for predicting deep endometrial invasion..$^{22,33}$ However, a retrospective study found that with regard to the prediction of deep myometrial invasion, DCE-MRI did not improve diagnostic performance when viewed independently with $\mathrm{T} 2 \mathrm{~W}$ images. ${ }^{23}$ In addition, since DCE-MRI is
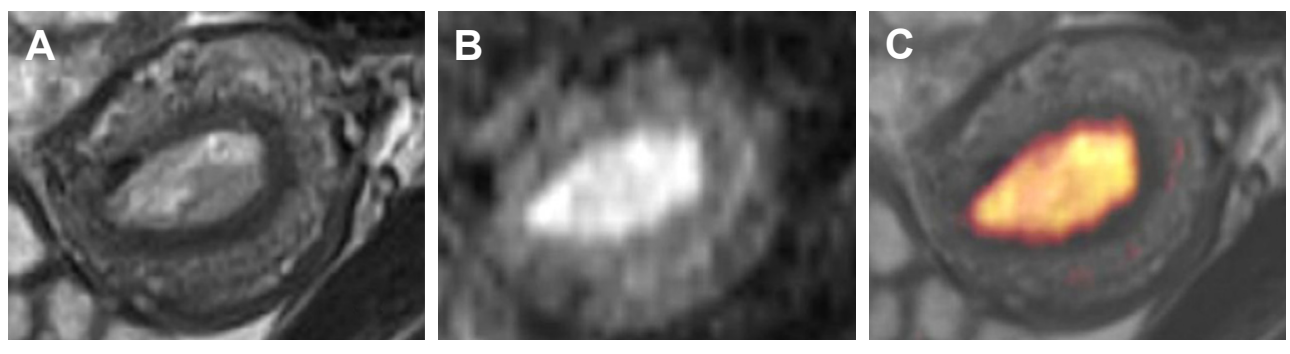

Figure I Magnetic resonance images of an endometrial cancer patient (stage IA).

Notes: (A) T2-weighted image shows irregular endometrial thickening and appears as heterogeneous signals. (B) DW image shows a lesion with a high signal. (C) Fused T2WI-DWI image shows clear anatomical structures and the lesion shows good contrast from the surrounding normal tissue.

Abbreviations: DWI, diffusion-weighted imaging; T2WI, T2-weighted imaging. 

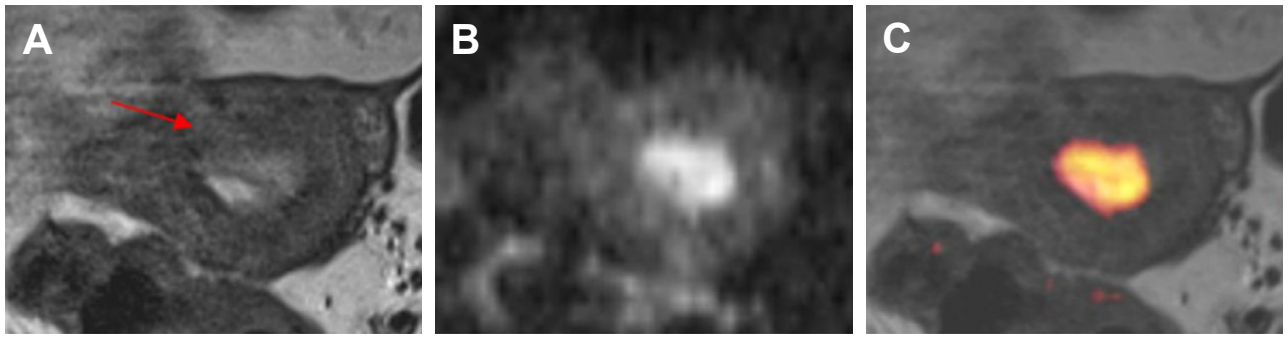

Figure 2 A 56-year-old woman with endometrial carcinoma (pathologic stage is IA).

Notes: (A) T2-weighted image shows that the myometrial signal was heterogeneous (arrow), and we judged the depth of myometrial invasion to be greater than one-half of the myometrial thickness, classifying this case stage as stage IB using T2WI. (B) DW image shows that the extent of the lesion was limited. (C) Fused T2WI-DWI image shows clear margins of the tumor and depth of invasion to be less than one-half of the myometrial thickness.

Abbreviations: DWI, diffusion-weighted imaging; T2WI, T2-weighted imaging.

performed using a contrast agent, it involves greater risk and financial cost to the patient.

Noninvasive DWI depicts differential molecular diffusion movements of water within various tissues. With DWI, increased cellularity and fewer mesenchymal structures between the glands in endometrial carcinomas may restrict water diffusion when compared with a normal endometrium. ${ }^{25,34}$ Endometrial cancer is observed as hyperintense areas that are depicted as hypointense on the apparent diffusion coefficient map. ${ }^{35}$ Several research groups have analyzed the usefulness of DWI in the detection and assessment of myometrial invasion in endometrial cancer. ${ }^{26,36}$ Researchers have reported that staging errors were excluded when incorporating DWI, and conventional MRI based on the fusion of DWI lesions for endometrial cancer is more sensitive for the effective assessment of lesions. ${ }^{34}$

Fused T2WI-DWI image is objective because it fuses morphologic and functional images to improve the anatomical localization of lesions in DWI. The anatomy is often not visualized clearly, and this may predispose it to the misdiagnosis of small lesions.$^{37}$ However, our 3T MRI scan shows higher signal-to-noise ratio and soft tissue resolution than low-field intensity MRI, which can then resolve this problem. The entire processing time for the fusion operation is $<0.5 \mathrm{~min}$. Also, the use of fused images has many other advantages, such as simplicity of operation, intuitiveness of images, noninvasiveness, and repeatability. Fused T2-weighted and DW images have been used in many other abdominal and pelvic tumor studies as well. ${ }^{27-30}$ Results have shown that fused images are very good for displaying anatomical structures and can provide functional information, thereby improving the accuracy rate of diagnosis.

There are several limitations to our study. First, a very limited number of cases were included in the current study; therefore, investigations with larger sample sizes and multicenter studies need to be conducted in the future. Second, we only included a few patients with advanced stage (IV) cancer because most of the patients were diagnosed and treated at earlier stages, with the fast development of medical science and financial conditions. Third, most patients with advanced stage cancer underwent radiochemotherapy without having the opportunity for surgical treatment. These limitations made it difficult for us to retrieve the appropriate pathologic samples and results that we required, which are very important in measuring the accuracy rates for stage IV patients.
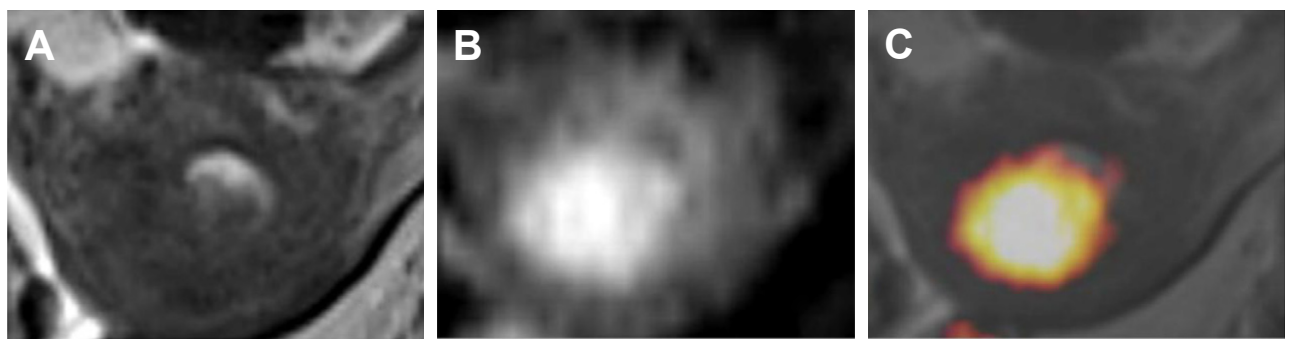

Figure 3 A 60-year-old woman with endometrial carcinoma (pathologic stage, IB).

Notes: (A) It was difficult to judge the depth of myometrial invasion with the T2-weighted image. (B) DW image shows the great extent of the high signal lesion. (C) Fused T2WI-DWI image shows clear margins of the tumor, and the depth of myometrial invasion to be greater than one-half of the myometrial thickness; the diagnosis was clearly stage IB.

Abbreviations: DWI, diffusion-weighted imaging; T2WI, T2-weighted imaging. 
Table 4 Diagnostic accuracy rate of endometrial carcinoma staging with T2WI and T2WI-DWI

\begin{tabular}{lllll}
\hline Diagnosis & Concordance & Discordance & Accuracy (\%) & R-value \\
\hline T2WI & 47 & 11 & $81.0(47 / 58)$ & 0.601 \\
T2WI-DWI & 55 & 3 & $94.8(55 / 58)$ & 0.023 \\
\hline
\end{tabular}

Abbreviations: DWI, diffusion-weighted magnetic resonance imaging; T2WI, T2-weighted magnetic resonance imaging.

\section{Conclusion}

The fusion of T2WI and DWI exhibited potential advantages in the noninvasive evaluation of invasion depth and preoperative staging. As an alternative approach to diagnosing endometrial carcinoma, this method reduces the cost and does not require the use of a contrast agent.

\section{Acknowledgment}

We would like to thank Professor Shengyong Wu, Dr Noel, and Dr Merry Shrestha for their assistance. We also appreciate research funding from the National Natural Science Foundation of China (No 81471761 and No 81501568), and the Tianjin Health and Family Planning Commission Science and Research Fund (No 2015KY08). We would like to thank LetPub (www.letpub.com) for providing linguistic assistance during the preparation of this manuscript.

\section{Disclosure}

The authors report no conflicts of interest in this work.

\section{References}

1. Jemal A, Siegel R, Xu J, Ward E. Cancer statistics, 2010. CA Cancer J Clin. 2010;60(5):277-300.

2. Sorosky JI. Endometrial cancer. Obstet Gynecol. 2012;120(2 Pt 1): 383-397.

3. Zhang H, Cui J, Jia L, et al. Comparison of laparoscopy and laparotomy for endometrial cancer. Int J Gynaecol Obstet. 2012;116(3):185-191.

4. Hahn HS, Yoon SG, Hong JS, et al. Conservative treatment with progestin and pregnancy outcomes in endometrial cancer. Int J Gynecol Cancer. 2009;19(6):1068-1073.

5. Aloisi A, Plotti F, Scaletta G, et al. Chemotherapy as adjuvant treatment for intermediate-high risk early-stage endometrial cancer: a pilot study. Int J Gynecol Cancer. 2015;25(8):1418-1423.

6. Amant F, Moerman P, Neven P, et al. Endometrial cancer. Lancet. 2005;366(9484):491-505.

7. Burke WM, Orr J, Leitao M, et al; Group SGOCPECW. Endometrial cancer: a review and current management strategies: part I. Gynecol Oncol. 2014;134(2):385-392.

8. Angioli R, Plotti F, Cafa EV, et al. Quality of life in patients with endometrial cancer treated with or without systematic lymphadenectomy. Eur J Obstet Gynecol Reprod Biol. 2013;170(2):539-543.

9. Capriglione S, Plotti F, Miranda A, et al. Further insight into prognostic factors in endometrial cancer: the new serum biomarker HE4. Expert Rev Anticancer Ther. 2017;17(1):9-18.

10. Haldorsen IS, Salvesen HB. Staging of endometrial carcinomas with MRI using traditional and novel MRI techniques. Clin Radiol. 2012; 67(1):2-12.

11. Chen T, Jansen L, Gondos A, et al. Survival of endometrial cancer patients in Germany in the early 21 st century: a period analysis by age, histology, and stage. BMC Cancer. 2012;12(1):128.
12. Benedetti Panici P, Basile S, Maneschi F, et al. Systematic pelvic lymphadenectomy vs. no lymphadenectomy in early-stage endometrial carcinoma: randomized clinical trial. J Natl Cancer Inst. 2008; 100(23):1707-1716.

13. Pecorelli S. Revised FIGO staging for carcinoma of the vulva, cervix, and endometrium. Int J Gynaecol Obstet. 2009;105(2):103-104.

14. Beddy P, O'Neill AC, Yamamoto AK, et al. FIGO staging system for endometrial cancer: added benefits of MR imaging. Radiographics. 2012;32(1):241-254.

15. Angioli R, Plotti F, Capriglione S, et al. Preoperative local staging of endometrial cancer: the challenge of imaging techniques and serum biomarkers. Arch Gynecol Obstet. 2016;294(6):1291-1298.

16. Masroor I, Zeeshan M, Afzal S, Ahmad N, Shafqat G. Diffusion weighted MR imaging (DWI) and ADC values in endometrial carcinoma. J Coll Physicians Surg Pak. 2010;20(11):709-713.

17. Kaneda S, Fujii S, Fukunaga T, et al. Myometrial invasion by endometrial carcinoma: evaluation with 3.0T MR imaging. Abdom Imaging. 2011;36(5):612-618.

18. Patel S, Liyanage SH, Sahdev A, Rockall AG, Reznek RH. Imaging of endometrial and cervical cancer. Insights Imaging. 2010;1(5-6):309-328.

19. Hori M, Kim T, Onishi H, et al. Endometrial cancer: preoperative staging using three-dimensional T2-weighted turbo spin-echo and diffusionweighted MR imaging at $3.0 \mathrm{~T}$ : a prospective comparative study. Eur Radiol. 2013;23(8):2296-2305.

20. Sala E, Crawford R, Senior E, et al. Added value of dynamic contrastenhanced magnetic resonance imaging in predicting advanced stage disease in patients with endometrial carcinoma. Int J Gynecol Cancer. 2009; 19(1):141-146.

21. Nakao Y, Yokoyama M, Hara K, et al. MR imaging in endometrial carcinoma as a diagnostic tool for the absence of myometrial invasion. Gynecol Oncol. 2006;102(2):343-347.

22. Manfredi R, Mirk P, Maresca G, et al. Local-regional staging of endometrial carcinoma: role of MR imaging in surgical planning. Radiology. 2004;231(2):372-378.

23. Rockall AG, Meroni R, Sohaib SA, et al. Evaluation of endometrial carcinoma on magnetic resonance imaging. Int J Gynecol Cancer. 2007;17(1):188-196.

24. Beddy P, Moyle P, Kataoka M, et al. Evaluation of depth of myometrial invasion and overall staging in endometrial cancer: comparison of diffusion-weighted and dynamic contrast-enhanced MR imaging. Radiology. 2012;262(2):530-537.

25. Seo JM, Kim CK, Choi D, Kwan Park B. Endometrial cancer: utility of diffusion-weighted magnetic resonance imaging with background body signal suppression at 3T. J Magn Reson Imaging. 2013;37(5):1151-1159.

26. Andreano A, Rechichi G, Rebora P, et al. MR diffusion imaging for preoperative staging of myometrial invasion in patients with endometrial cancer: a systematic review and meta-analysis. Eur Radiol. 2014; 24(6):1327-1338.

27. Nishie A, Stolpen AH, Obuchi M, et al. Evaluation of locally recurrent pelvic malignancy: performance of T2- and diffusion-weighted MRI with image fusion. J Magn Reson Imaging. 2008;28(3):705-713.

28. Tsushima Y, Takano A, Taketomi-Takahashi A, Endo K. Body diffusion-weighted MR imaging using high $b$-value for malignant tumor screening: usefulness and necessity of referring to T2-weighted images and creating fusion images. Acad Radiol. 2007;14(6):643-650.

29. Rosenkrantz AB, Mannelli L, Kong X, et al. Prostate cancer: utility of fusion of T2-weighted and high $\mathrm{b}$-value diffusion-weighted images for peripheral zone tumor detection and localization. J Magn Reson Imaging. 2011;34(1):95-100. 
30. Brenner R, Metens T, Bali M, Demetter P, Matos C. Pancreatic neuroendocrine tumor: added value of fusion of T2-weighted imaging and high b-value diffusion-weighted imaging for tumor detection. Eur $J$ Radiol. 2012;81(5):e746-e749.

31. Dogan D, Inan N, Sarisoy HT, et al. Preoperative evaluation of myometrial invasion in endometrial carcinoma: diagnostic performance of 3T MRI. Abdom Imaging. 2013;38(2):388-396.

32. Wu LM, Xu JR, Gu HY, et al. Predictive value of T2-weighted imaging and contrast-enhanced MR imaging in assessing myometrial invasion in endometrial cancer: a pooled analysis of prospective studies. Eur Radiol. 2013;23(2):435-449.

33. Saez F, Urresola A, Larena JA, et al. Endometrial carcinoma: assessment of myometrial invasion with plain and gadoliniumenhanced MR imaging. J Magn Reson Imaging. 2000;12(3): $460-466$.
34. Emlik D, Kiresi D, Ozdemir S, Celik C, Karakose S. Preoperative assessment of myometrial and cervical invasion in endometrial carcinoma: comparison of multi-section dynamic MR imaging using a three dimensional FLASH technique and T2-weighted MR imaging. $J$ Med Imaging Radiat Oncol. 2010;54(3):202-210.

35. Gallego JC, Porta A, Pardo MC, Fernandez C. Evaluation of myometrial invasion in endometrial cancer: comparison of diffusion-weighted magnetic resonance and intraoperative frozen sections. Abdom Imaging. 2014;39(5):1021-1026.

36. Rechichi G, Galimberti S, Signorelli M, et al. Myometrial invasion in endometrial cancer: diagnostic performance of diffusion-weighted MR imaging at 1.5-T. Eur Radiol. 2010;20(3):754-762.

37. Torricelli P, Ferraresi S, Fiocchi F, et al. 3-T MRI in the preoperative evaluation of depth of myometrial infiltration in endometrial cancer. AJR Am J Roentgenol. 2008;190(2):489-495.

\section{Publish your work in this journal}

OncoTargets and Therapy is an international, peer-reviewed, open access journal focusing on the pathological basis of all cancers, potential targets for therapy and treatment protocols employed to improve the management of cancer patients. The journal also focuses on the impact of management programs and new therapeutic agents and protocols on

\section{Dovepress}

patient perspectives such as quality of life, adherence and satisfaction. The manuscript management system is completely online and includes a very quick and fair peer-review system, which is all easy to use. Visit http://www.dovepress.com/testimonials.php to read real quotes from published authors.

Submit your manuscript here: http://www.dovepress.com/oncotargets-and-therapy-journal 